\title{
ANÁLISE COMPARATIVA DA ARTROPOFAUNA DE SOLO EM ÁREAS DE CULTIVO AGROECOLÓGICO, CONVENCIONAL E FRAGMENTO DE FLORESTA NO MUNICÍPIO DE VALENÇA - BA
}

\author{
COMPARATIVE ANALYSIS OF SOIL ARTHROPOD FAUNA IN AREAS OF \\ AGROECOLOGICAL, CONVENTIONAL AND FOREST FRAGMENT IN THE CITY OF \\ VALENÇA - BA
}

\begin{abstract}
ANÁLISIS COMPARATIVO DE LA ARTROPOFAUNA DE SUELO EN ÁREAS DE CULTIVO AGROECOLÓGICO, CONVENCIONAL Y FRAGMENTO FORESTAL EN EL MUNICIPIO

DE VALENÇA - BA
\end{abstract}

\author{
Marília Mascarenhas Lima ${ }^{1}$ \\ Ailana Cerqueira da Silva ${ }^{2}$ \\ Pedro Henrique Barbosa Ferreira ${ }^{3}$
}

\begin{abstract}
Resumo
Considerando o papel dos artrópodes na execução de diversos processos ecológicos, entende-se que seja relevante conhecer a funcionalidade desses organismos e a complexidade ecológica dessas comunidades em áreas de importância econômica ou ecológica. Nesse sentido, esta pesquisa de campo objetivou comparar a composição da fauna de artrópodes edáficos em três diferentes áreas rurais do município de Valença no estado da Bahia: fragmento florestal, vitrine de fruteiras e sistema agroflorestal convencional. Três coletas desses organismos foram realizadas por meio de oito armadilhas do tipo "pitfall" em cada tratamento. Em cada armadilha uma solução de álcool a 50\% foi utilizada, para preservação do material biológico. Os artrópodes de solo extraídos das armadilhas foram acondicionados em álcool a 70\%. Em seguida, foram levados para o laboratório de biologia do IFBaiano para posterior triagem ao menor nível taxonômico possível com auxílio de microscópio estereoscópico. Após coleta, triagem e identificação dos indivíduos em laboratório, foram obtidos os índices de diversidade, equitabilidade e riqueza de grupos, para posterior comparação dos dados analisados nos diferentes ambientes estudados. Um total de 4.732 indivíduos foi encontrado, distribuídos nas classes Insecta, Entognatha, Malacostraca, Arachnida, Diplopoda e Chilopoda. Os valores de riqueza de espécies, bem como os índices de diversidade oscilaram entre os diferentes tratamentos. Diferentemente, os valores de abundância foram consideravelmente maiores na vitrine de fruteiras, o que se explica pela presença marcante das formigas. Os resultados obtidos denotam que, apesar da intensa redução e fragmentação do habitat, a mata mantém uma considerável diversidade de artrópodes de solo.
\end{abstract}

Palavras-chave: Artrópodes. Diversidade. Pitfall.

\begin{abstract}
Considering the role of arthropods in the execution of various ecological processes, it is understood that it is relevant to know the functionality of these organisms and the ecological complexity of these communities in areas of economic or ecological importance. In this sense, this field research aimed to compare the fauna composition of edaphic arthropods in three different rural areas of the municipality of Valença in the state of Bahia: forest fragment, fruit showcase and conventional agroforestry system. Three collections of these organisms were performed using eight pitfall traps in each treatment. In each trap, a 50\% alcohol solution was used to preserve biological material. The soil arthropods extracted from the traps were conditioned in $70 \%$ alcohol. Then, they were taken to the IFBaiano Biology laboratory for further screening at the lowest possible taxonomic level with the aid of a stereoscopic microscope. After collection, screening and identification of individuals in the laboratory, the indices of diversity, equitability and richness of groups were obtained, for later

\footnotetext{
${ }^{1}$ Mestre em Genética, Biodiversidade e Conservação pela Universidade Estadual do Sudoeste da Bahia; analista ambiental pela prefeitura municipal de Vitória da Conquista. E-mail: lilamascarenhas@hotmail.com.

${ }^{2}$ Técnica em agroecologia pelo IFBaiano - campus Valença. E-mail: ailana.10cerqueira@gmail.com.

${ }^{3}$ Técnica em agroecologia pelo IFBaiano - campus Valença. E-mail: pferrera319@gmail.com.
} 
comparison of the data analyzed in the different environments studied. A total of 4,732 individuals were found, distributed in the classes Insecta, Entognatha, Malacostraca, Arachnida, Diplopoda and Chilopoda. The species richness values, as well as the diversity indexes fluctuated between the different treatments. In contrast, the abundance values were considerably higher in the fruit showcase, which is explained by the marked presence of the ants. The results obtained show that, despite the intense reduction and fragmentation of the habitat, the forest maintains a considerable diversity of soil arthropods.

Keywords: Arthropods. Diversity. Pitfall.

\section{Resumen}

Considerándose el rol de los artrópodos en la ejecución de diversos procesos ecológicos, se entiende que sea relevante conocer la funcionalidad de esos organismos y la complejidad ecológica de esas comunidades en áreas de importancia económica o ecológica. En ese sentido, esta investigación de campo tuvo el objetivo de comparar la composición de la fauna de artrópodos edáficos en tres áreas rurales del municipio de Valença, en el estado de Bahia: fragmento forestal, área de frutales y sistema agroforestal convencional. Se realizaron tres recolecciones de esos organismos por medio de trampas de tipo "pitfall" en cada tratamiento. En cada trampa, se utilizó una solución de alcohol al 50\%, para la preservación del material biológico. Los artrópodos de suelo extraídos de las trampas fueron puestos en alcohol al 70\%. Luego, fueron llevados al laboratorio de biología del IFBahiano para posterior triaje al más pequeño nivel taxonómico posible, con auxilio de microscopio estereoscópico. Después de la recolección, triaje e identificación de los individuos en laboratorio, se obtuvieron los índices de diversidad, ecuabilidad y riqueza de grupos, para posterior comparación de los datos analizados en los diferentes ambientes estudiados. Se encontró un total de 4.732 individuos, distribuidos en las clases Insecta, Entognatha, Malacostraca, Arachnida, Diplopoda y Chilopoda. Los valores de riqueza de especies, así como los índices de diversidad oscilaron entre los distintos tratamientos. Por otro lado, los valores de abundancia fueron considerablemente más significativos en el área de frutales, lo que se explica por la presencia importante de hormigas. Los resultados obtenidos demuestran que, pese a la intensa reducción y fragmentación del hábitat, la mata mantiene una diversidad considerable de artrópodos en el suelo.

Palabras-clave: Artrópodos. Diversidad. Pitfall.

\section{Introdução}

Grande parte dos táxons de Invertebrados está representada no solo (CORREIA; OLIVEIRA, 2000). Entre eles, os artrópodes de solo, os quais têm papel fundamental em diversos processos ecológicos, como a decomposição da matéria orgânica, pois influenciam em diferentes graus a ciclagem de nutrientes, aeração e fertilidade do solo (AMARAL; SANTOS, 2015; LEMOS et al., 2016; SEFFRIN; COSTA; DEQUECH, 2005; SILVA et al., 2016). Outras espécies se enquadram como pragas de cultivos por se alimentarem de sementes, raízes, folhas ou outras partes de plantas, prejudicando ou impossibilitando o seu desenvolvimento (AMORIM et al., 2013).

A fauna edáfica em ecossistemas ou agroecossistemas pode indicar o nível de degradação, recuperação ou conservação do solo, pois esses organismos são sensíveis a alterações antrópicas da cobertura vegetal, aspecto que pode incidir em sua densidade e diversidade (LUCENA et al., 2017). Algumas práticas agriculturais, como a aplicação de pesticidas, podem perturbar a estrutura do solo como habitat, reduzindo a diversidade e abundância de espécies benéficas (CORREIA; OLIVEIRA, 2000). Portanto, conhecer a funcionalidade desses organismos e a complexidade ecológica de tais comunidades em áreas 
de importância econômica ou ecológica é de grande relevância.

O presente estudo teve como objetivo comparar a composição da artropodofauna de solo em três diferentes áreas: fragmento florestal, vitrine de fruteiras e sistema agroflorestal convencional, utilizando-se medidas de abundância, riqueza, diversidade e equitabilidade de artrópodes edáficos em cada um dos ambientes.

\section{Fundamentação teórica}

As características do ambiente afetam a diversidade de invertebrados do solo. Ambientes com maior riqueza de espécies vegetais tendem a apresentar maiores riquezas de fauna edáfica e equilíbrio ambiental. Assim, o tipo de manejo e de cobertura vegetal tem influência sobre a riqueza e abundância da mesofauna e da macrofauna do solo (AMARAL; SANTOS, 2015). De acordo com Silva et al. (2006), as comunidades da macrofauna edáfica em ambientes de vegetação nativa são mais estáveis; de modo semelhante, sistemas submetidos a manejo dos solos mais conservacionistas têm sido menos afetadas (SILVA et al., 2006).

Algumas ordens de artrópodes desempenham papel de destaque com relação aos benefícios que podem trazer ao solo. Os coleópteros apresentam muitos representantes com potencial para atuar no controle biológico, compondo um dos principais grupos de predadores em ambiente edáfico (LEMOS et al., 2016) e apresentam grande frequência em sistemas de cultivo de sorgo (SEFFRIN; COSTA; DEQUECH, 2005), pastagens (AMORIM et al., 2013) e florestas (BATTIROLA et al., 2015). Hymenoptera é uma ordem relatada por contribuir para o aumento da infiltração da água, favorecendo a manutenção do ambiente saudável (SILVA et al., 2016); é principalmente representada pela família Formicidae, muito abundante em áreas agrícolas (AMARAL; SANTOS, 2015; ROSSI et al., 2009; LEMOS et al., 2016; SILVA et al., 2012). As aranhas são também frequentemente encontradas neste tipo de ambiente, atuando como importantes predadoras de inimigos naturais (AMARAL; SANTOS, 2015; LEMOS et al., 2016).

Estudos sobre a composição da mesofauna de solo em área sob manejo agroecológico encontraram, entre os diversos táxons possíveis, os Acari (ROSSI et al., 2009), Coleoptera e Orthoptera (SILVA et al., 2012) de modo mais significativo. Por outro lado, os ortópteros da família Grillydae são abundantes em áreas de pastagens, podendo indicar intensa perturbação ambiental (AMORIM et al., 2013).

A utilização de armadilhas para capturar artrópodes é comum em estudos de 
diversidade e composição de comunidades. Técnicas passivas de captura de artrópodes são consideradas vantajosas por reduzirem o tempo de coleta de campo, a possibilidade de interferência relacionada à experiência do coletor, além de serem simples e de baixo custo. As armadilhas de queda do tipo "pit-fall traps" são recomendadas para captura de artrópodes edáficos (AQUINO; AGUIAR-MENENEZ; QUEIROZ, 2006).

O uso de insetos considerados indicadores ecológicos por Silveira Neto et al. (1995) permitiram uma avaliação do impacto ambiental, indicando interferência das mudanças do ambiente sobre a comunidade estudada. Candido et al., (2012), a partir de índices ecológicos, encontraram em seu estudo uma maior diversidade em ambiente conservado (mata ciliar) quando comparado à área em recuperação. Dessa forma, a comunidade da macrofauna edáfica pode servir como bioindicadora em distintos tipos de sistema de produção, de maneira que se pode usá-los para determinar alternativas de manejo sustentável em sistemas agropecuários (SILVA et al., 2006).

\section{Metodologia}

O local estudado situa-se na área rural do município de Valença no estado da Bahia, a qual está destinada para aulas práticas e pesquisas de campo dos cursos oferecidos pelo IFBaiano, campus Valença (Figura 1). Denominada Fazenda Aldeia, esse espaço tem uma área total de 33,77 hectares, sendo que mais da metade é destinada a uso de cultivos e benfeitorias. O restante dessa área, cerca de 15,52 ha, enquadra-se em área de preservação permanente (APP) e reserva legal.

Três ambientes foram selecionados para compor diferentes tratamentos nesse estudo, que permitiram a sua comparação por meio de índices ecológicos: remanescente de floresta, sistema agroflorestal convencional e uma vitrine de fruteiras. O remanescente de floresta caracteriza-se por estar em estágio intermediário de regeneração; trata-se de mata ciliar que circunda o leito do rio Piau (Área de Preservação Permanente - APP). A Vitrine de Fruteiras possui uma área de 0,7 hectares, (coordenadas 13.343517 - 39.124292). Nesse espaço são cultivadas diversas culturas como: manga, cupuaçu, laranja, tangerina, banana, limão, umbu, fruta-pão, acerola, goiaba, cacau, cajá, graviola e maracujá. A lavoura se dá a pleno sol e o manejo inclui o uso de fertilizantes químicos. 
Figura 1: Área de estudo com demarcações dos ambientes selecionados para coleta

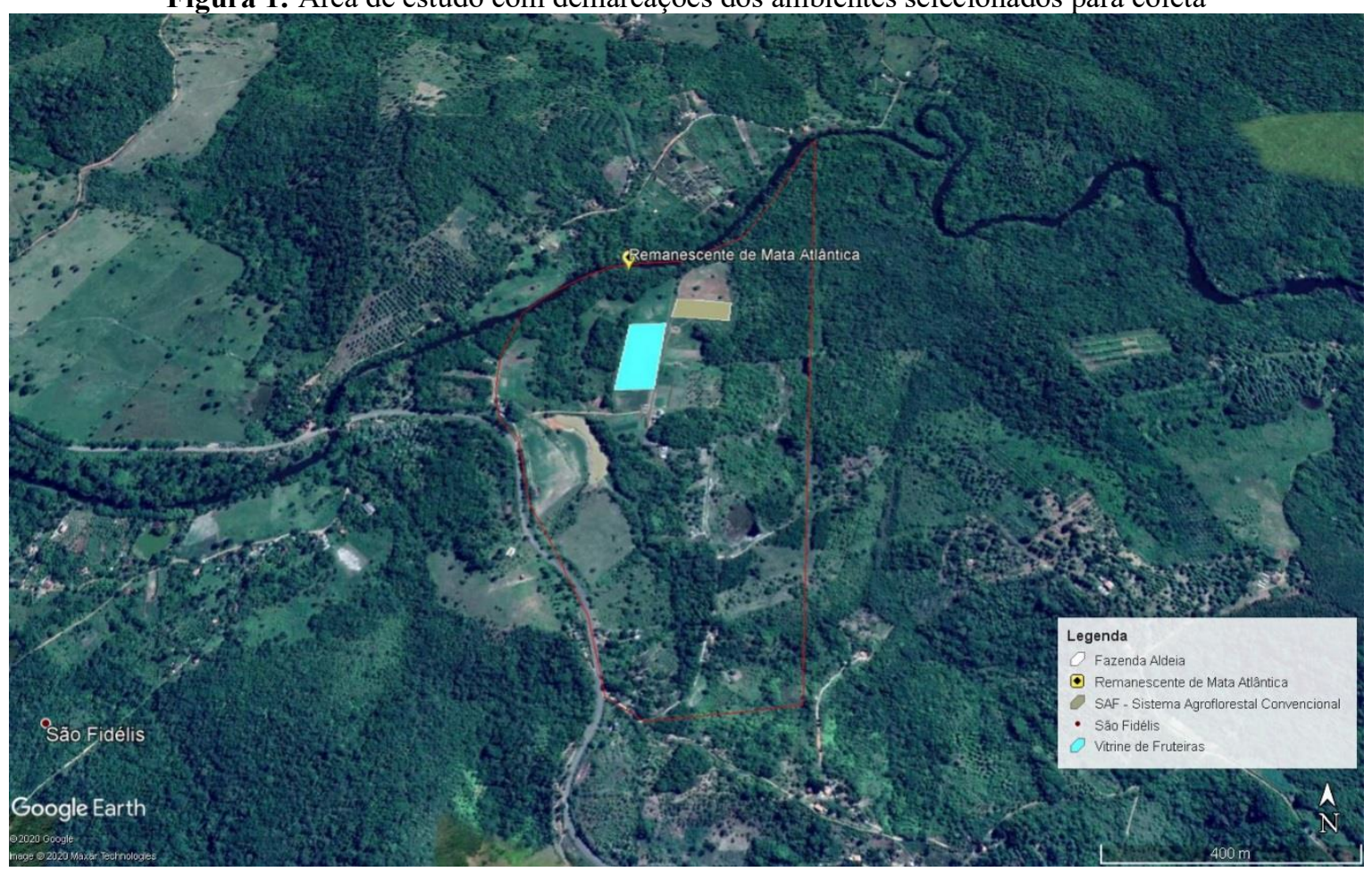

Fonte: Google Earth

O Sistema Agroflorestal (SAF) Convencional possui uma área total de 0,6 hectares, com localização nas coordenadas geográficas 13.341887 - 39.123231. Quatro diferentes plantios foram implantados: seringueira, cacau, banana e mandioca. Esse sistema requer condições específicas de implantação. O cacau inicialmente deverá ser sombreado pela bananeira e mandioca. Após esses cultivos serem colhidos, a seringueira fará o sombreamento.

As ferramentas de captura de artrópodes de solo utilizadas foram as armadilhas pitfall, as quais foram instaladas em 2 grupos com 4 armadilhas dispostas em forma de Y em cada um dos 3 ambientes a serem amostrados, totalizando 24 armadilhas por coleta. Respeitou-se uma distância das bordas de no mínimo $35 \mathrm{~m}$ para evitar-se o "efeito de borda" no fragmento de floresta. Os pratos descartáveis presos com palitos de madeira serviram para impedir que águas pluviais transbordassem a solução acondicionada dentro das armadilhas (Figura 2). As armadilhas ficaram em campo por 3 dias consecutivos. 


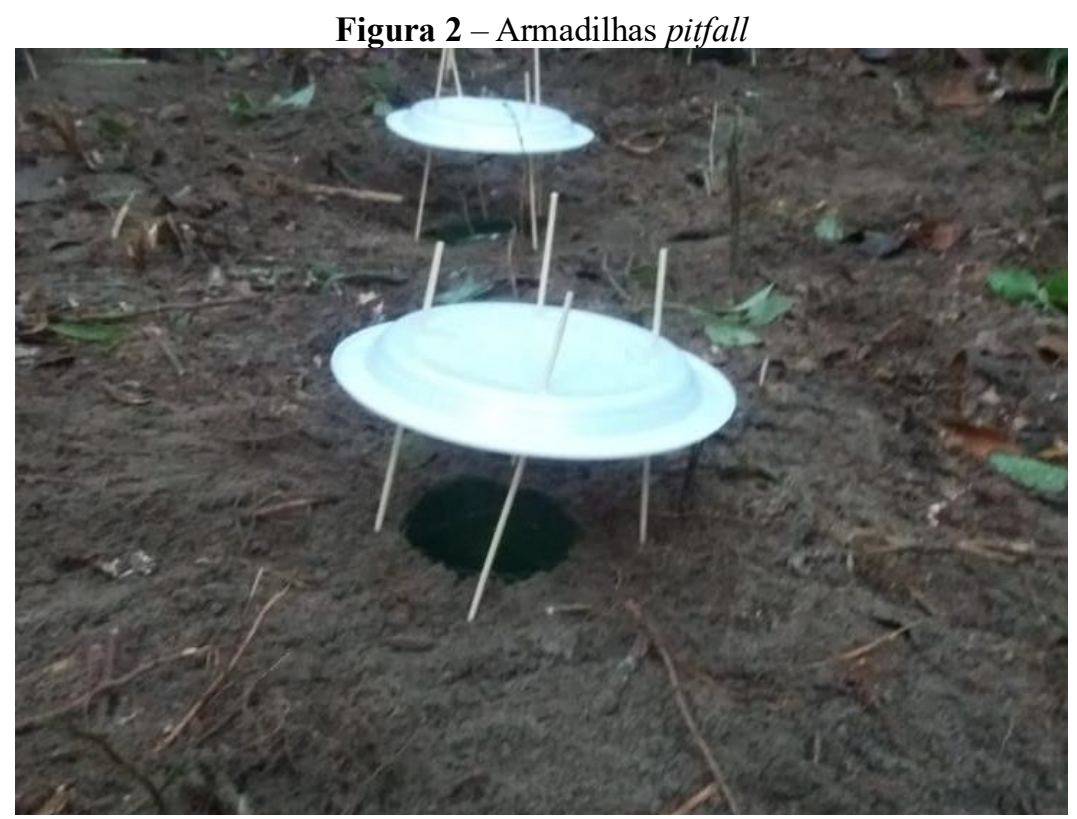

Fonte: própria (2018)

\section{Desenvolvimento}

Três coletas realizaram-se entre os meses de maio a novembro de 2018. Cada armadilha continha uma solução de álcool a 50\%, para preservação do material biológico. Os artrópodes de solo extraídos das armadilhas foram acondicionados em álcool a 70\% para sua preservação. Em seguida, foram levados ao laboratório de biologia do IFBaiano, campus Valença, para posterior triagem ao menor nível taxonômico possível, com auxílio de microscópio estereoscópico binocular desenvolvido pela Toya, utilizando-se as objetivas de 2x e 4x ou lupa de mesa com aumento de 3x da Solver HL-200LED (Figura 3).

Após a coleta, triagem e identificação dos indivíduos, obtiveram-se as medidas de Diversidade de Simpson, Diversidade de Shannon, Equabilidade de Pielou e riqueza de grupos, para posterior comparação dos dados analisados nos diferentes ambientes estudados. O número de unidades taxonômicas, seja classe, ordem ou família, foi tratado como riqueza, enquanto que o número de indivíduos, como abundância. 


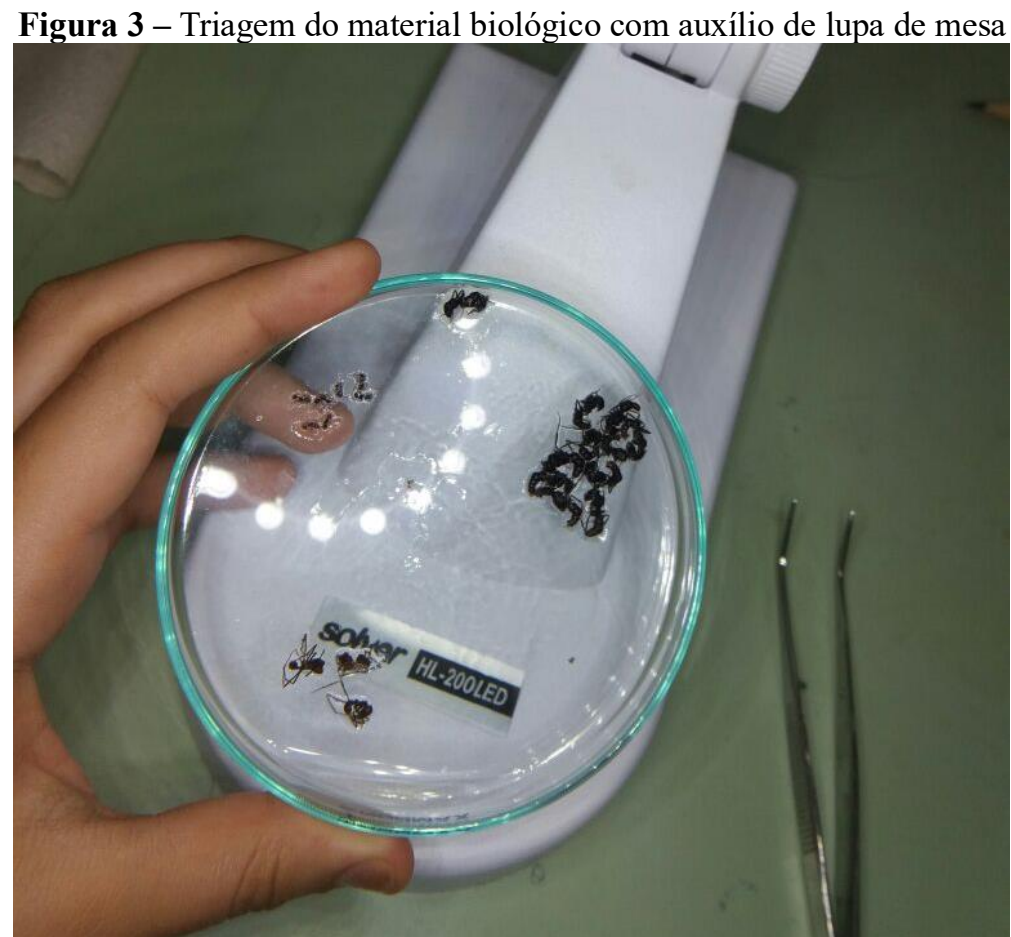

Fonte: própria (2018)

\section{Resultados e discussão}

Um total de 4.732 indivíduos foram encontrados, distribuídos nas classes Insecta, Entognatha, Malacostraca, Arachnida, Diplopoda e Chilopoda. As ordens da classe Insecta foram representadas por Hymenoptera $(65,97 \%)$, Coleoptera $(14,49 \%)$, Diptera $(7,98 \%)$, Hemiptera $(1,77 \%)$, Orthoptera $(1,28 \%)$, Lepidoptera $(0,08 \%)$, Isoptera $(0,06 \%)$ e Dermaptera $(0,06 \%)$.

Os Entognathas foram representados pelas ordens Collembola (2,57\%) e Diplura $(0,23 \%)$. O sub-filo Malacostraca foi representado pela ordem Isopoda $(0,16 \%)$, enquanto que Arachnida por Araneae (4,24\%), Mesostignata (0,02\%) e Opilione (0,19\%). As classes Diplopoda e Chilopoda não tiveram nesse estudo as ordens identificadas.

Os valores de riqueza de espécies (Gráfico 1) e os índices de diversidade oscilaram entre os diferentes tratamentos, no entanto, conforme observado nos Índices de Simpson e de Shannon (Tabela 1), esses valores foram sempre altos no fragmento de floresta e no SAF. Diferentemente, os valores de abundância foram consideravelmente maiores na vitrine de fruteiras, o que se explica pela presença marcante das formigas (Gráfico 2). 
Gráfico 1 - Valores de riqueza nos diferentes tratamentos

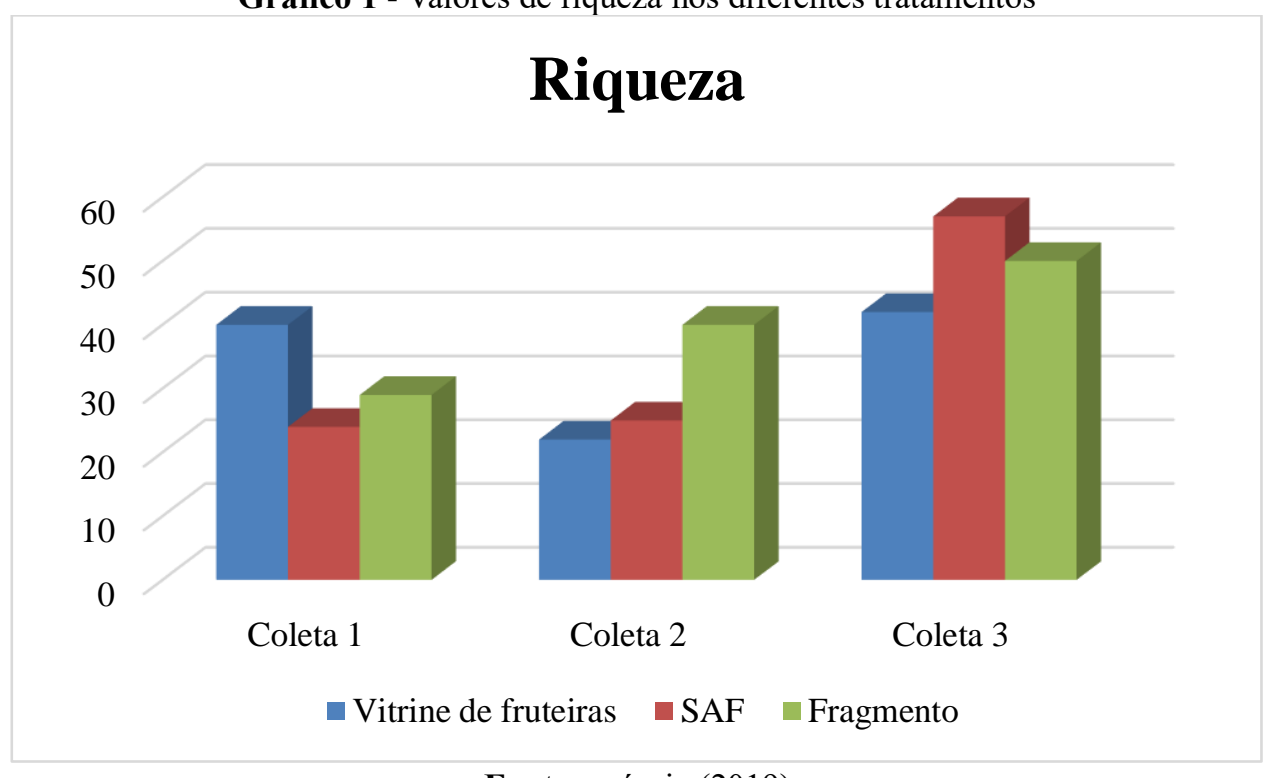

Fonte: própria (2019)

Gráfico 2 - Valores de abundância nos diferentes tratamentos

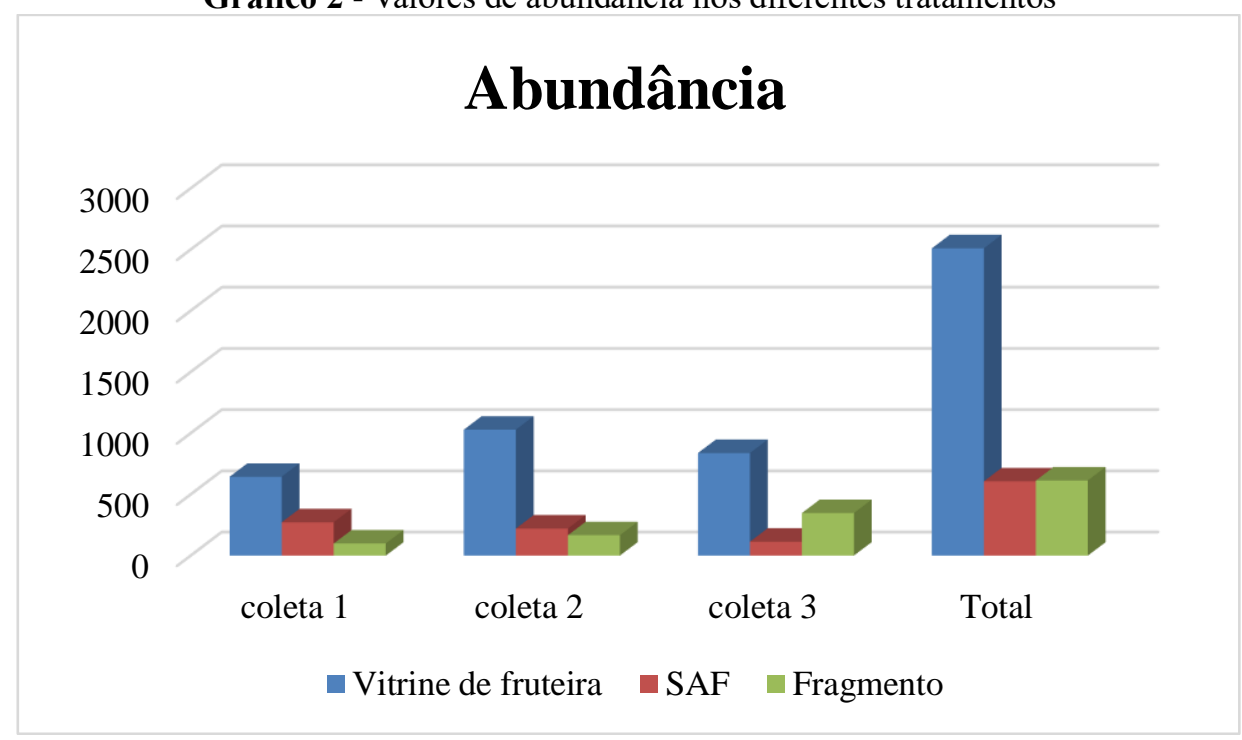

Fonte: própria (2019)

Os valores obtidos a partir dos índices de Diversidade de Simpson, Shannon e Equitabilidade de Pielou estão demonstrados na Tabela 1. Os índices de Equabilidade de Pielou foram mais altos no fragmento de floresta quando comparados aos demais ambientes, o que era esperado, pois ambientes naturais e com altos índices de diversidade tendem a apresentar maior uniformidade na distribuição das espécies. 
Tabela 1 - índices de diversidade de Simpson, Shannon e Equabilidade de Pielou nos diferentes tratamentos

\begin{tabular}{|c|c|c|c|}
\hline Coleta 1 & $\begin{array}{l}\text { Vitrine de } \\
\text { fruteiras }\end{array}$ & $\begin{array}{c}\text { SAF } \\
\text { Convencional } \\
\end{array}$ & $\begin{array}{c}\text { Fragmento de } \\
\text { floresta }\end{array}$ \\
\hline Índice de Diversidade de Simpson (D) & 0,81 & 0,84 & 0,89 \\
\hline Índice de Diversidade de Shannon $(\mathrm{H})$ & 2,24 & 2,24 & 2,75 \\
\hline Índice de equabilidade de Pielou $(\mathrm{J})$ & 0,61 & 0,7 & 0,82 \\
\hline Coleta 2 & $\begin{array}{l}\begin{array}{l}\text { Vitrine de } \\
\text { fruteiras }\end{array} \\
\end{array}$ & $\begin{array}{c}\text { SAF } \\
\text { Convencional } \\
\end{array}$ & $\begin{array}{c}\text { Fragmento de } \\
\text { floresta }\end{array}$ \\
\hline Índice de Diversidade de Simpson (D) & 0,29 & 0,8 & 0,82 \\
\hline Índice de Diversidade de Shannon $(\mathrm{H})$ & 0,8 & 2,12 & 2,49 \\
\hline Índice de equabilidade de Pielou $(\mathrm{J})$ & 0,26 & 0,66 & 0,68 \\
\hline Coleta 3 & $\begin{array}{l}\text { Vitrine de } \\
\text { fruteiras }\end{array}$ & $\begin{array}{c}\text { SAF } \\
\text { Convencional } \\
\end{array}$ & $\begin{array}{c}\text { Fragmento de } \\
\text { floresta }\end{array}$ \\
\hline Índice de Diversidade de Simpson (D) & 0,76 & 0,72 & 0,87 \\
\hline Índice de Diversidade de Shannon $(\mathrm{H})$ & 1,95 & 1,89 & 2,34 \\
\hline Índice de equabilidade de Pielou $(\mathrm{J})$ & 0,52 & 0,47 & 0,7 \\
\hline
\end{tabular}

Fonte: própria (2019)

As ordens que predominaram em frequência absoluta, ou seja, a porcentagem (ou proporção) de ocorrência de uma espécie em uma determinada área, foram Hymenoptera, Coleoptera e Diptera. Essas três ordens somaram 88,44\% dos indivíduos coletados. Tacca, Klein e Preuss (2017), ao compararem a artropodofauna de solo de uma monocultura de eucalipto com um remanescente de Floresta Atlântica, encontraram entre as ordens predominantes Hymenoptera (32,9\%), seguidas de Collembola $(22,4 \%)$ e Diptera $(13,9 \%)$, somando $69,2 \%$ do total de indivíduos coletados nos dois ambientes.

Hymenoptera tem sido representada principalmente pela família Formicidae que apresenta grande abundância, sobretudo em áreas agrícolas em estudos de artropodofauna utilizando armadilhas de queda (AMARAL; SANTOS, 2015; ROSSI et al., 2009; LEMOS et al., 2016; SILVA et al., 2012). As formigas foram bem representadas nos três tratamentos, porém sua abundância foi alta no SAF e principalmente na vitrine de fruteiras, elevando sobremaneira esses valores. Esse resultado era esperado, visto que esse grupo também pode ser considerado praga e causar enormes prejuízos na lavoura.

Os formicídeos totalizaram 2.545 indivíduos $(53,78 \%)$ do número total de indivíduos coletados. O percentual de formigas no SAF foi de $71,56 \%$, enquanto na vitrine essa família representou 54,08\% e na floresta apenas $6,03 \%$ dos indivíduos coletados nesse ambiente. A presença marcante desse grupo taxonômico no SAF e na vitrine de fruteiras pode sugerir desequilíbrio nestes ambientes (MELLO et al., 2016). Outros estudos tiveram resultados similares apresentando predominância de formigas, sobretudo em áreas agrícolas (AMARAL; SANTOS, 2015; SILVA et al., 2012).

Ao analisar a assembleia de formigas entre diferentes ambientes, Braga et al. (2010) 
encontraram uma similaridade na composição de espécies dessa família em agroecossistema eucaliptal quando comparados aos sistemas florestais em diferentes estágios sucessionais, diferentemente do ambiente de pastagem que apresentou menor similaridade. Observou-se no estudo supracitado um gradiente na frequência de espécies tipicamente de florestas entre as áreas estudadas, no qual a floresta primária encontra-se em um extremo, apresentando alta frequência, e a pastagem no outro, com reduzida incidência dessas espécies. Assim, pode-se concluir que o tipo de cobertura vegetal e o uso e manejo do solo possivelmente influenciam na estrutura da comunidade.

Aranhas são também frequentemente encontradas em sistemas agrícolas, atuando como importantes predadores de inimigos naturais (AMARAL; SANTOS, 2015; LEMOS et al., 2016). Os predadores, tais como aranhas e besouros, são comumente generalistas. Atacam variedades de presas, em diferentes estágios de desenvolvimento no ciclo de vida. Podem se alimentar de pragas em produções agrícolas, e quando não conseguem atingir e manter os baixos níveis causadores de prejuízo econômico, eles diminuem a sua densidade populacional (AMARAL, 2011). As aranhas obtiveram uma frequência significativa no total amostrado, e estiveram presentes nos três tratamentos.

Os colêmbolos são minúsculos insetos saltadores que têm como base alimentícia fungos, plantas jovens e sementes. São importantes, pois suas fezes são ricas em nutrientes, que são disponibilizados para as plantas através da decomposição (AMARAL, 2011). Eles estiveram presentes no fragmento de floresta e no sistema agroflorestal. Provavelmente, sua abundância foi subestimada devido ao seu tamanho reduzido.

\section{Considerações finais}

Os índices de diversidade foram altos no fragmento de floresta e no sistema agroflorestal; já na vitrine de fruteiras apresentaram variações. Os Índices de Equabilidade de Pielou foram mais altos no fragmento de floresta quando comparados aos demais ambientes, pois ambientes naturais e com altos índices de diversidade tendem a apresentar maior uniformidade na distribuição das espécies. Com relação à abundância, os valores foram consideravelmente maiores nas áreas agrícolas devido à presença marcante das formigas.

Os resultados obtidos denotam que, apesar do pequeno tamanho do fragmento e do intenso grau de fragmentação, a mata mantém uma considerável diversidade de artrópodes de solo. Apesar de ser manejado com utilização reduzida de fertilizantes químicos e herbicidas, o SAF também apresentou alto índice de diversidade de artrópodes do solo quando comparado 
com a vitrine de fruteiras, sobretudo na segunda coleta. Pesquisas adicionais explorando o objeto de estudo, seriam fundamentais para melhor compreensão da composição da artropodofauna em solos com diferentes formas de uso.

\section{Referências}

ABREU, R.L.R.; LIMA, S.S.; OLIVEIRA, N.C.R.; LEITE, L.F.C. Fauna edáfica sob diferentes níveis de palhada em cultivo de cana-de-açúcar. Pesq. Agropec. Trop., Goiânia, v. 44, n. 4, p. 409-416, out./dez. 2014.

AMARAL, A. A. Fundamentos de Agroecologia. Curitiba: Livro Técnico, 2011. 160 p.

AMARAL, A.A.; SANTOS, G.M. Artrópodes do solo em áreas antrópicas com diferentes coberturas vegetais. In: Enciclopédia Biosfera, v. 11, n. 22, p. 62-71. Goiânia: Centro Científico Conhecer, 2015.

AMORIM, I.A.A.; AQUINO, A.L.; SILVA, E.M.J.; MATOS, T.E.S.; SILVA, T.P.; RODRIGUES, D.M. Levantamento de artrópodes da superfície do solo em área de pastagem no assentamento alegria, Marabá-PA. Agroecossistemas, [S.l.], v. 5, n. 1, p. 62-67, 2013.

AQUINO, A.M.; AGUIAR-MENEZES, E.L.; QUEIROZ, J.M. Recomendações para coleta de artrópodes terrestres por Armadilhas de Queda ("Pitfall-Traps"). Seropédica: Embrapa Agrobiologia, 2006. (Série Embrapa Agrobiologia. Circular técnica, 18)

BATTIROLA, L.D.; SILVA, L.S. ALMEIDA, F.M.; BATISTELLA, D.A.; PENA-BARBOA, J.P.P.; CHAGAS JR., A. BRESCOVIT, A.D. Artrópodes de solo. In: RODRIGUES, D.J.; NORONHA, J.C.; VINDICA, V.F.; BARBOSA, F.R. Biodiversidade do Parque Estadual Cristalino. Sinop (MT): Áttema Editorial, 2015. 284 p.

BRAGA. D. L. et al. Avaliação rápida da diversidade de formigas em sistemas de uso do solo no sul da Bahia. Neotropical Entomology. Londrina, v. 39, n. 4, p. 464-469, ago. 2010.

CANDIDO; A.K.A.A.; SILVA, N.M.; BARBOSA, D.S.; FARIAS, L.N.; SOUZA, W.P. Fauna edáfica como biodindicadores de qualidade ambiental na nascente do Rio São Lourenço, Campo Verde-MT, Brasil. Engenharia Ambiental, Espírito Santo do Pinhal, v. 9, n. 1, p. 067-082, jan. /mar.2012

CORREIA, M.E.F.; OLIVEIRA, L.C.M. de. Fauna de solo: aspectos gerais e metodológicos. Seropédica: Embrapa Agrobiologia, fev. 2000. 46p. (Embrapa Agrobiologia. Documentos, 112).

LEMOS, W.P.; SANTO, L.N.E.; LIMA, M.M.; OLIVEIRA, T.C. Inimigos naturais de solo em sistemas de cultivo de palma de óleo (Elaeis guineensis) no município de Tomé-Açu, PA. Cadernos de Agroecologia, [S.l.], v. 10, n. 3, maio 2016. ISSN 2236-7934.

LUCENA, E.O. et al. O solo como ecossistema. In: SOUZA, T.A.F. \& SANTOS, D. (org.). Solos em sistemas agroecológicos. 1. ed. Areia: Edição dos Autores, 2017. cap. 1, p. 10-45. 
MELLO, E. R. et al. Formas de controle das formigas. Viçosa, MG: UFV, 2016. 21 p.

ROSSI, C.; NOBRE, C.; COELHO, C.; BENAZZI, E.; RODRIGUES, K.; CORREIA, M.E. Efeito de diferentes coberturas vegetais sobre a mesofauna edáfica em manejo agroecológico. Rev. Bras. de Agroecologia, Dois Vizinhos, v. 4, n. 2, nov., 2009.

SEFFRIN, R.C.A.S.; COSTA, E.C.; DEQUECH, S.T.B. Artropodofauna do solo em sistemas direto e convencional de cultivo de sorgo (Sorghum bicolor (L.) Moench). Ciênc. agrotec., Lavras, v. 30, n. 4, p. 597-602, jul./ago., 2006.

SILVA, R.F.; AQUINO, A.M.; MERCANTE F.M.; GUIMARÃES, M.F. Macrofauna invertebrada do solo sob diferentes sistemas de produção em Latossolo da Região do Cerrado. Pesq. agropec. bras., Brasília, v. 41, n. 4, p. 697-704, abr., 2006.

SILVA, M.R.A.; JORDÃO, B.A.; COSTA, J.; RODRIGUES, F.C.; SOUZA, E.M. BRITO, E.S.G. Levantamento entomofaunístico em áreas de cultivo agroecológico e convencional no município de Ouricuri - PE. In: CONGRESSO NORTE NORDESTE DE PESQUISA E INOVAÇÃO - CONNEPI, 7., 2012, Palmas. Anais [...]. Palmas TO: IFTO, 2012.

SILVA, M.C.S.; SOBRINHO, F.S.L.; SILVA, A.V.; BARROS, R.P. A utilização de armadilhas tipo pitfall para levantamento da fauna edáfico em solos com cultivo de Manihot esculenta Crantz. e Opuntia spp. na zona rural de Arapiraca-AL. In: CONGRESSO NORDESTINO DE BIÓlOGOS - CONGREBIO, 6., 2016, João Pessoa. Anais [...]. João Pessoa: Universidade Federal da Paraíba, 2016.

SILVEIRA NETO, S.; MONTEIRO, R.C.; ZUCCHI, R.A.; MORAES, R.C.B. Uso da análise faunística de insetos na avaliação do impacto ambiental. Sci. Agric., Piracicaba, v. 52, n. 1, p. 9-15, jan./abr., 1995.

TACCA, D.; KLEIN, C.; PREUSS, J.F. Artropodofauna do solo em um bosque de eucalipto e um remanescente de mata nativa no sul do Brasil. Revista Thema, [S.l.], v. 14, n. 2, p. 249$261,2017$. 